\title{
Measuring the strength of dissipative inflation
}

\author{
Luis Herrera*, Alicia Di Prisco ${ }^{\dagger}$ \\ Area de Física Teórica. Facultad de Ciencias. \\ Universidad de Salamanca. 37008 Salamanca, Spain. \\ and \\ Diego Pavón ${ }^{\ddagger}$ \\ Departamento de Física, Facultad de Ciencias, \\ Edificio Cc, Universidad Autónoma de Barcelona, \\ 08193 Bellaterra, Spain.
}

\begin{abstract}
We comment on recently proposed dissipative inflationary models. It is shown that the strength of the inflationary expansion is related to a specific combination of thermodynamic variables which is known to measure the instability of self-gravitating dissipative systems.
\end{abstract}

\footnotetext{
*Also at UCV, Caracas, Venezuela; E-mail address: lherrera@gugu.usal.es ${ }^{\dagger}$ On leave from Universidad Central de Venezuela, Caracas, Venezuela

${ }^{\ddagger}$ E-mail address: diego@ulises.uab.es
} 
In recent years different authors have considered the possibility that inflation could be driven by dissipative fluid effects (see [1] - [6] and references therein), or somehow equivalently by particle production as a result of the interaction between the quantum vacuum and the gravitational field. Recently the comparison between the cosmological consequences of both processes has been studied in detail [7]. A phase of accelerated expansion shortly after the big-bang has been invoked many times as a mechanism able to get rid of the horizon and flatness problems that beset the standard cosmological model [8].

It is the purpose of this note to relate the "strength" of the inflationary phase of those models to a given parameter formed by a specific combination of hydrodynamical variables. This parameter has been shown to affect critically the evolution of self-gravitating dissipative objects [9]-[11].

The study of the departure of dissipative systems from hydrostatic equilibrium has shown that the aforesaid parameter $\alpha$ enters the equation of motion of any fluid element in such a way that the inertial mass density term is multiplied by a factor that vanishes for a certain value of that parameter indicating therefore the existence of a critical point. In general, the "effective" inertial mass density decreases with the increasing of $\alpha$. In some cases (pure shear or bulk viscosity [9]) the critical point is well beyond the border where the causality requirements are violated. In others (pure thermal conduction [10, 11]) the latter requirements are violated slightly below the critical point. However, in the general case (heat conduction plus viscosity)

$$
\alpha=\frac{1}{(\rho+p)}\left(\frac{\zeta}{2 \tau_{\zeta}}+\frac{\kappa T}{\tau_{\kappa}}+\frac{2 \eta}{3 \tau_{\eta}}\right),
$$

it appears that causality may break down beyond the critical point [9]. In the above expression $\rho$ and $p$ are the energy density and equilibrium pressure of the fluid, respectively; $\zeta, \kappa$ and $\eta$ denote the transport coefficients of bulk viscosity, heat conduction and shear viscosity, respectively; and the three different $\tau$ stand for the corresponding relaxation times. Finally $T$ indicates the fluid temperature. Here we shall not consider anisotropic cosmological models and so the shear coefficient will not appear.

Our motivation is two fold: on one hand we wish to delve deeper into the physical meaning of $\alpha$ and, on the other hand, we would like to provide a "control" parameter for the strength of expansion in any given inflationary model driven by dissipative processes. 
We begin by considering a model, recently proposed by Maartens and collaborators [6] of an inflationary solution with causal heat flux. They start from the inhomogenous shear-free model of Modak which in comoving coordinates has the line element [12], [13]

$$
d s^{2}=-\left[1+M(t) r^{2}\right]^{2} d t^{2}+a^{2}(t)\left[d r^{2}+r^{2}\left(d \theta^{2}+\sin ^{2} \theta d \phi^{2}\right)\right] .
$$

Next, they assume that $M=M_{0}$ and $H \equiv \dot{a} / a=H_{0}$, are both positive constants and that the stress-energy tensor of the fluid can be written as

$$
T_{a b}=(\rho+p) u_{a} u_{b}+p g_{a b}+2 q_{(a} u_{b)},
$$

where $q_{a}=(q / a) \delta^{1}{ }_{a}, q$ being a covariant scalar measure of the energy-flux magnitude. Then the expressions for the matter variables which follow from field equations are

$$
\begin{gathered}
\rho=\frac{3 H_{0}^{2}}{\left(1+M_{0} r^{2}\right)^{2}}, \\
p=\left[\frac{4 M_{0}}{a_{0}^{2}\left(1+M_{0} r^{2}\right)}\right] e^{-2 H_{0} t}-\rho, \\
q=-\left[\frac{4 M_{0} H_{0} r}{a_{0}\left(1+M_{0} r^{2}\right)^{2}}\right] e^{-H_{0} t},
\end{gathered}
$$

where the fact that $a=a_{0} e^{H_{0} t}$ was used. Also, in oder to satisfy the IsraelStewart causal transport equation [14], [15] they assume

$$
\begin{gathered}
\tau_{\kappa}=\left(1+M_{0} r^{2}\right) H_{0}^{-1} \\
T=\frac{U(t)}{1+M_{0} r^{2}} \quad(U(t)>0) .
\end{gathered}
$$

In the model of Maartens et al. T decreases radially outward notwithstanding the heat-flux is directed inward. This is so because the thermal effect concomitant to the fluid acceleration $\dot{u}_{\alpha}=2 M_{0} r\left(1+M_{0} r^{2}\right)^{-1} \delta^{1}{ }_{a}$ dominates over the temperature gradient [16].

By virtue of (4), (5), (7) and (8), equation (1) becomes into

$$
\alpha=\left(\frac{\kappa U(t)}{12}\right)\left(\frac{\Theta}{M_{0}}\right) a_{0}^{2} e^{2 H_{0} t},
$$


where the fluid expansion rate is given by

$$
\Theta \equiv u_{; a}^{a}=\frac{3 H_{0}}{1+M_{0} r^{2}}
$$

Now, it is clear that for larger values of $a_{0}$ and $H_{0}$, and smaller values of $M_{0}$ (which implies larger values of $\Theta$ ), the inflationary expansion will be stronger. On the other hand, as immediately follows from (9), it also implies larger values of $\alpha$. Therefore, stronger inflationary expansions are related to larger values of $\alpha$ (for fixed $U(t)$ ). This result reinforces the physical meaning of $\alpha$ as a measure of the instability of the system (see [9]-[11], [17], [18]). All this suggests that $\alpha$ can be used as a "control parameter" of the strength of expansion in a given inflationary model driven by causal heat flux.

As we shall see the previous comment about the potential role of $\alpha$ may be extended to inflationary models driven by viscous stresses with no heat fluxes. Indeed, in the case of inflation driven by bulk viscosity the generated entropy may be written as (see equation (38) in [5] प)

$$
S \approx \frac{4 \alpha-1}{2 \alpha}
$$

where $\alpha$ is now given by (11) specialized to the $\kappa=\eta=0$ case [9]. Equation (11) shows that the generation of entropy increases with $\alpha$. Also observe that in the family of models presented in [3], it appears that

$$
\alpha=\frac{1}{2 \gamma}
$$

where as usual $\gamma$ is the adiabatic index and enters the equation of state of the fluid

$$
p=(\gamma-1) \rho .
$$

Thus for the case of ultrarelativistic particles $(\gamma=4 / 3)$ we have $\alpha=3 / 8$, which is rather near to the limiting value $\alpha=1 / 2$. In other words, large values of $\alpha$ are expected in typical inflationary scenarios driven by bulk viscosity.

Closely related to the bulk viscous pressure is the phenomenon of particle production since the latter can be phenomelogically interpreted as bulk

\footnotetext{
${ }^{1}$ Our $\alpha$ should not be counfused with theirs.
} 
viscosity [19], [20] and therefore our above result involving $\pi$ finds a natural extension to inflationary models driven either by cosmological particle production (due to the interaction between the gravitational field and the quatum vacuum) or by the decay of massive particles of the primordial plasma into lighter ones. In particular there is a well-known relationship between this dissipative pressure and the rate of particle production $\Gamma$ for isotropic cosmological expansions when the former is assumed to be adiabatic [7]

$$
\pi=-(\rho+p) \frac{\Gamma}{3 H} \text {. }
$$

In this connection Pavón et al. [21] have considered the decay of a nonrelativistic fluid of massive particles with energy density $\rho_{1}$ into a radiationlike fluid, with energy density $\rho_{2}$ proportional to the fourth power of temperature. Then assuming the relaxation time to be of the order of magnitude of the mean free interaction time between the matter and radiation particles, it follows that

$$
\alpha=\frac{\beta}{2\left(\frac{4}{3}+\frac{\rho_{1}}{\rho_{2}}\right)}
$$

(note that our $\beta$ corresponds to their $\alpha$ ).

From (15) we have that $\alpha$ grows with $\beta$, i.e. with the bulk viscosity pressure, thereby larger values of $\alpha$ are related to stronger expansions. This is only natural since for expanding universes the latter pressure implies a negative contribution to the total fluid pressure and therefore helps to accelerate the expansion.

Our next exemple includes two family of models proposed by Barrow 22 based in an effective bulk viscous pressure related to the very high rate of quantum production of fundamental strings [23]. These two families correspond to string-driven inflationary and deflationary models depending on if the Universe evolves from a non-inflationary stage to an inflationary one or from a de Sitter phase to a Friedmann expansion, respectively. Barrow assumes a spatially-flat scenario governed by

$$
3 H^{2}=\rho,
$$

and with the viscous pressure given by $\pi=-3 \zeta H$. For $\zeta \propto \rho^{m}$ ( $m=$ constant), Barrow obtains when $m<1 / 2$ and $H \geq H_{0}=$ constant, isotropic cosmological models which exhibit inflationary behaviour. They begin at a Friedmann singularity and approach from above a de Sitter state as $t \rightarrow \infty$. 
If $m>1 / 2$ and $H \leq H_{0}$ deflationary expansions follow. These begin in a de Sitter state with $H=H_{0}$ and evolve towards a Friedmann asymptote. In both kind of models one has $\dot{H}<0$.

Now, assuming as in 21] $\tau_{\zeta} \propto H^{-1}$ and for $\zeta$ the power-law dependence upon the density mentioned above, it is easily obtained from (1) with $\kappa=$ $\eta=0$

$$
\alpha \propto H^{2 m-1}
$$

where (13) has been used.

Thus, for $m>1 / 2, \alpha$ decreases during the expansion, explaining thereby the deflationary behaviour of the models. For $m<1 / 2, \alpha$ increases as $H$ decreases, leading to stronger expansion (inflation). This suggests that any inflationary model (driven by dissipation) endowed with a mechanism for achieving an exit from inflation (a desirable feature), should allow for changing from an increasing to a decreasing $\alpha$, during the evolution. All these results confirm the role of $\alpha$ as a measure of the "strength" of the expansion mentioned above.

It is worth stressing again that in the more general case (heat flux plus shear and bulk viscosity), $\alpha$ may be larger than unity without violating causality conditions [9]. This suggests that one may build more "efficient" inflationary models by resorting to a combination of all forms of dissipation (heat current plus either viscosity or particle production or both). Obviously, the difficulty there would be to deal with a more general geometry since to accommodate an energy-flux together with shear stress the spacetime must be both inhomogeneous and anisotropic.

Before closing we would like to emphasize that bulk viscosity may also be interpreted as the effect of some scalar field, say $\phi$, which has found a more ample audience as a candidate to drive the very early accelerated expansion invoked by inflationary models. The connection between these two disparate quantities, $\pi=-\Gamma \dot{\phi}^{2} /(3 H)$, follows from specializing equation (14) to the scalar field case only that in this instance $\Gamma$ is the decay rate of $\phi$. This has been used to phenomelogically model the reheating phase of the universe right after inflation [24]. Although the behaviour of the corresponding control parameter $\alpha$ may also be studied following parallel lines to that of above, the involved task is by no means trivial and we leave it to a future research. 


\section{Acknowledgements}

This work has been partially supported by the Spanish Ministry of Eductaion under grant PB94-0718.

\section{References}

[1] D. Pavón. J. Bafaluy and D. Jou (1991) Class. Quantum Grav. 8, 347.

[2] V. Romano and D. Pavón (1993) Phys.Rev. D 47, 1396.

[3] R. Maartens (1995) Class. Quantum Grav. 12, 1455.

[4] W. Zimdahl (1996) Phys. Rev. D 53, 5483.

[5] R. Maartens and V. Méndez (1997) Phys. Rev. D 55, 1937.

[6] R. Maartens, M. Govender and S. D. Maharaj (1999) Gen. Rel. Grav. 31, 816.

[7] W. Zimdahl (1999) Phys. Rev. D (in the press). Report astroph/9910483.

[8] E. W. Kolb and M. S. Turner (1990) The Early Universe (AddisonWesley, Redwood City).

[9] L. Herrera and J. Martínez (1998) Class. Quantum Grav. 15, 407.

[10] L. Herrera, A. Di Prisco, J. Hernández-Pastora, J. Martín and J. Martínez (1997) Class. Quantum Grav. 14, 2239.

[11] L. Herrera and J. Martínez (1997) Class. Quantum Grav. 14, 2697.

[12] B. Modak (1984) J. Astrophys. Astr. 5, 317.

[13] A. Krasiński (1997) Inhomogeneous cosmological models (CUP, Cambridge).

[14] W. Israel and J. Stewart (1979) Ann. Phys. (N.Y.) 118, 341. 
[15] D. Pavón, D. Jou and J. Casas-Vázquez (1982) Ann. Inst. H. Poincaré A 36, 79 .

[16] C.W. Misner, K. Thorne and J.A. Wheeler (1973) Gravitation (Freeman, New York).

[17] L. Herrera and J. Martínez (1998) Astr. Sp. Sci. 259, 235.

[18] L. Herrera and A. Di Prisco (1999) Gen. Relativ. Grav. 31, 301.

[19] B.L. Hu (1982) Phys. Lett. A 90, 375.

[20] Ya. B. Zeldovich (1970) Sov. Phys. JETP Lett. 12, 307.

[21] D. Pavón, J. Gariel and G. Le Denmat (1996) Gen. Relativ. Grav. 28, 573.

[22] J.D. Barrow, (1988) Nucl. Phys. B 310, 743.

[23] N. Turok, (1988) Phys. Rev. Lett. 60, 549.

[24] W. Zimdahl and D. Pavón (1994) Mont. Not. R. Astr. Soc. 266, 872. 